\title{
Veterinary Education in the Role of Geometric Imagination in Imagen-Ology: Three-Dimensional Mental Configurations of Two-Dimensional Radiologic Plates
}

\author{
Rodríguez-Salazar LM${ }^{1 *}$, Oliver González MR $^{2}$ and García Tovar CG \\ ${ }^{1}$ Instituto Politécnico Nacional, Mexico
}

${ }^{2}$ Departamento de Ciencias Biológicas, UNAM, Mexico

*Corresponding author: Luis Mauricio Rodríguez Salazar, Research Professor in the Master of Science Program in Methodology of Science in the Research Center in Economic, Management and Social Sciences, National Polytechnic Institute, Mexico

Submission: 海 May 20, 2018; Published: 海 September 18, 2018

\begin{abstract}
The main aim of our research group in epistemology of imagination-a theoretical proposal by the first author of this paper, is shift the veterinary education in imagen-ology, grounded in the representation of anatomical structures to be interpreted by students, toward cognitive actions to in which students can constructs their own representations. In short, move from representations for student's interpretation, toward cognitive representative actions where the students construct the radiologic plates. Our proposal for the construction of representative actions entails the imagination of actions grounded in geometrical thinking, the representative actions must be grounded in imaginary actions, then, we change the concept of imaging in radiology, for to imagen-ology in imagination.
\end{abstract}

Keywords: Veterinary; Education; Epistemology; Imagination; Imagen ology; Radiology; Geometric think; Geometric imagination; Mental configurations

\section{Introduction}

The role of geometric think is the construction of mental configurations of geometric images of anatomical structures of the animal body serving for the spatial location, as well as for the establish their relationships with the different regions and cavities of animal's body. Hence, the aim goal of education is to encourage the process for which the student can imagine the things that he cannot see. In anatomy, by means of the dissection, the student can see structures like muscles, bones and the organs of the body, but in a living animal, he cannot see it. Then, the role of geometric imagination in imagen-ology is the construction of threedimensional mental configurations due that animal anatomy is three-dimensional, and the radiologic plates are two-dimensional. We need mental constructions of three-dimensional representaction of the radiological plates as near as possible to our threedimensional configuration achieved throughout imagine-action.

Epistemology of imagination in imagen-ology by radiology

In radiology, the proposal of the epistemology of imagination is the construction of three-dimensional (3D) mental images by mean of two-dimensional (2D) image of the radiologic plates, passing from the interpretation of radiologic plates, to the construction of them by the students. We need then give to students the technical and theoretical foundations about the construction of radiologic plates, in which is recorded the shadow of the anatomical structure of the body. From epistemological point of view, this process takes us to remind the allegory of Plato's Cave in which the people watch shadows of men and animals projected in a wall, used by Plato to compare the effect of education and the lack of it in our nature.

The epistemology of imagen-ology in Veterinary, understood as the relationship between student as subject and animal as object. The X-ray that go across the structures of animal with less impediment, is recorded in radiologic plate as radiolucent or black areas, while the areas that are not reached by X-ray show up as opaque or white areas [1,2]. So, what we observe in a radiologic plate is the shadow of the object structures reached by the X-ray, in analogy with the shadows by effect of the sunlight, as too in the allegory of Plato's Cave, as can be observed in panel A of the Figure 1. 


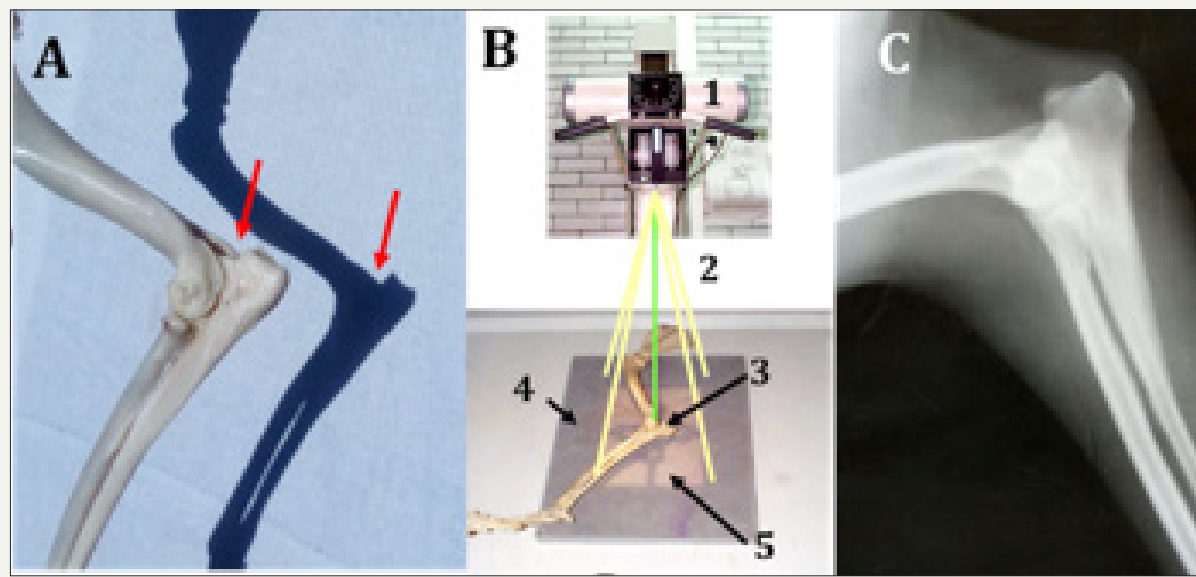

Figure 1: Shadows projected by sunlight (visible light) and X-rays (outside the spectrum of visible light).

For the creation of radiographs, it is necessary to play with the X-ray beam so that it penetrates in a certain direction and thus obtain a two-dimensional image, based on knowledge of the anatomy, creating the shadow left by each of the structures. Because the anatomical structures are three-dimensional, at least two orthogonal radiographic images corresponding to the $\mathrm{X}$ and Y Cartesian planes are required, which, when combined, allow us to mentally construct the three-dimensional configuration of the anatomical structure learned in the dissections. Even, in some cases, we can play with the direction of the rays and make oblique shots, which will generate a more complete three-dimensional mental image and not overlook any structure that overlaps with others by having only a two-dimensional plane $\mathrm{X}$ and $\mathrm{Y}$ obtained.

\section{Geometric imagination in radiology by teaching of medical imagen-ology}

In a recent work of our research group [3], reference was made to the role that geometric thought plays in the imaginary mental configuration of histological structures, as well as their relationships, with the Cartesian coordinates $\mathrm{X}$ and $\mathrm{Y}$ coming into play, which for three-dimensionality the Z-axis is also considered. As already mentioned, the images obtained by X-rays are twodimensional shadows generated when projecting the X-ray beam to specimen. A three-dimensional mental configuration based on a system of axes is required, of the $\mathrm{X}, \mathrm{Y}$ and $\mathrm{Z}$ coordinates represented in Figure 2, to obtain at least two orthogonal radiographs for threedimensionality. This way, the $\mathrm{X}$ axis goes from left to right, the $\mathrm{Y}$ axis from bottom to top and the $\mathrm{Z}$ axis from back to front [4]. Applying this in the abdominal cavity, in a lateral radiograph, Figure 3, the $\mathrm{X}$ axis would be long, from cranial to caudal, the $\mathrm{Y}$ axis would be wide, from ventral to dorsal, and the $\mathrm{Z}$ axis would be thickness from side to side of the cavity. Now, in a ventro-dorsal (VD) radiograph shown in Figure 4, the $\mathrm{X}$ axis is from side to side, the $\mathrm{Y}$ is caudal to cranial and the $\mathrm{Z}$ is from dorsal to ventral. Then, the positions are calculated by measuring the lateral position, height and depth for our spatial location.

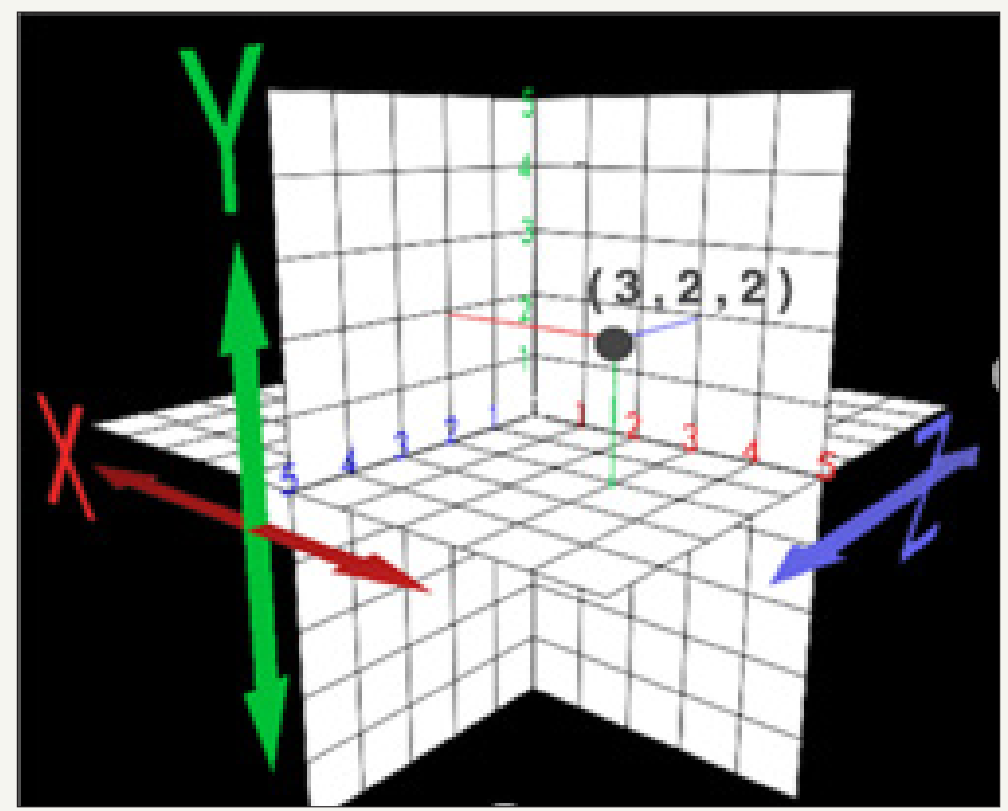

Figure 2: $X, Y$ and $Z$ axis system. 


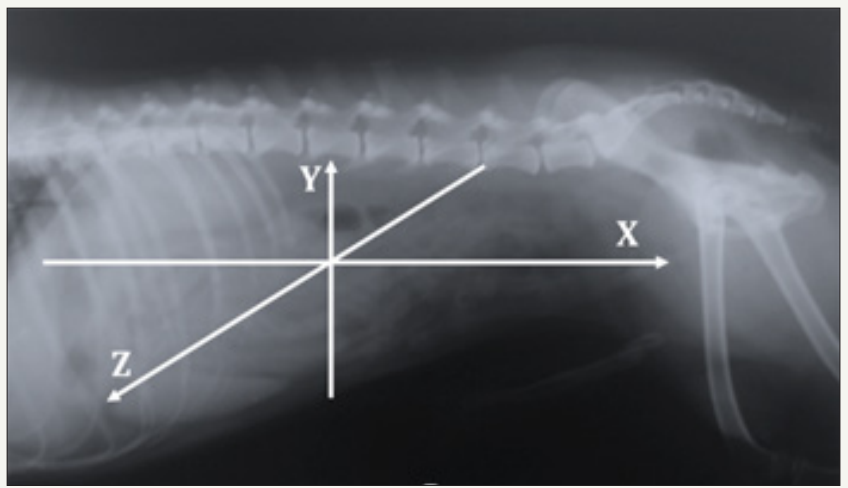

Figure 3: $\mathrm{X}, \mathrm{Y}$ and $\mathrm{Z}$ axis system applied to $\mathrm{L}$ abdominal radiograph.

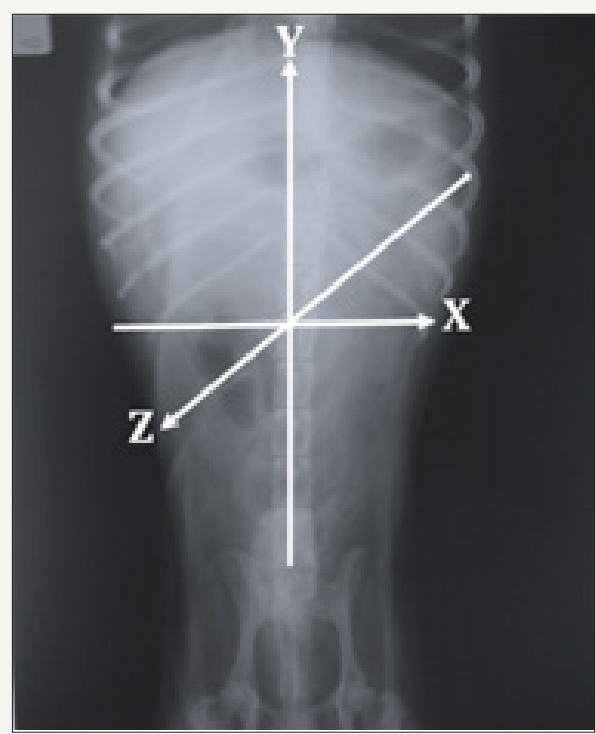

Figure 4: X, Y, Z axis system applied to a VD abdominal radiograph.

From the epistemology of the imagination and with the topographical approach, we can say that, within the cavities, the anatomical location of each structure or organ, as well as its relationships, is studied, taking this geometric thought to the areas of topographic anatomy. The student must understand the relationships, cranial, caudal, dorsal, ventral and lateral. In this way, having the mental configuration of the anatomical organization inside the cavities, supported by the geometric thought and using the three axes as mentioned, can be represented with some accuracy the place in the space in which each are located organs and their relationships. This imaginary configuration through geometrical thinking will allow the student to decide how to place the patient and what projection would give the X-ray beam to obtain the required radiographs and move from the interpretation of what is represented on the plates, to representations created by the own actions of the students. Transform representations into representactions, where the imagination turns into imaginary-actions.

\section{Imagine-actions and represent-actions in tridimensional mental configurations in radiology}

The proposal of the epistemology of imagination is that mental imaginary configurations becomes in real images in imagen-ology, throughout the imagination of actions that we call imagine-actions.
A practical example of this proposal is the mental configuration of organ location and their relationship with other organs contained in the abdominal cavity of the dog-especially gastrointestinal, taking into account their tridimensional structure grounded in anatomy previous knowledge, in order to locate, for example, ingested bodies, common in them. Thus, we know that the cecum and ascending colon are to the right of the median plane of the abdominal cavity and the descending colon is to the left, both connected with the transverse colon that goes from right to left [5-7]. Considering the nomenclature used to designate the radiographic images, in the LD projection in the abdominal cavity, despite the contrast medium applied -barium enema, cecum and ascendant colon cannot be distinguished of descendent colon, since these overlaps.

So, we don't know where an injury or a foreign body could be, if in the cecum, descending colon or ascending colon. Regarding the transverse colon [3] its travel from one side to another is not appreciated. For this, taking into account the technical recommendation of the orthogonal shots (having two shots in $90 \mathrm{c}$ ), in the VD projection of the abdomen and with the use of contrast medium, all its parts could be clearly distinguished: cecum, ascending colon, transverse colon and descending colon, whose numbering is equal to panel A. In addition, it would be possible to 
accurately determine its anatomical situation within the cavity and, therefore, where the possible injury could be.

One might think that with the VD projection it would be sufficient for this study of the large intestine, but this is not the case, since with this projection the location of the colon and rectum [5] in the dorsal to ventral direction could not be specified, as can be seen in Figure 4 is located at a medium height, discarding some constipation where the descending colon changes its position, with a displacement towards the ventral or an inflammation of lympho-nodes, among other anomalies. With the imagine-actions, it is possible to carry out in an appropriate way all the steps to follow for taking a radiographic plate, whatever the case, from how to place the patient (position), the intensity of the X-rays, time of exposure, $\mathrm{X}$ ray beam projection (alignment) and development process. In this way, all the actions carried out to create the representation of the reality expressed in the radiographic plates as it represents-actions [8], will have their origin in the imaginary action of the student or imagine-actions and would be guided by the exercise of the geometric thought, within the framework of the epistemology of the imagination. The names of the structures and organs mentioned in this work are based on the Nomina Anatomica Veterinaria [9].

\section{Conclusions}

The images obtained from the animal organism by means of X-rays, are similar to the shadows of the objects produced by the rays of visible light, in such a way that, in the teaching of radiological studies they must build in their mind, both the teacher as the student, the imaginary three-dimensional conformation of the organism to get to understand the two-dimensional images obtained in radiographic plates. From our epistemological proposal, geometric thought is very supportive, with which the Cartesian, $\mathrm{X}$ and $\mathrm{Y}$ axes are applied, as well as the axis of the $\mathrm{Z}$ that determines the depth of the bodies. Thus, the actions indicated in the processes and the techniques of radiological studies are carried out, that is to make the represents-actions that are determined by the imagineactions.

\section{Acknowledgement}

Project SIP: 20180338

Project PIAPIME ID 2.11.09.18 FES Cuautitlán UNAM.

Project DGAPA-UNAM PAPIME PE 205717.

\section{References}

1. Thrall DE (2003) Manual de diagnóstico radiológico veterinario. $\left(4^{\text {th }}\right.$ edn), Elsevier, Barcelona, Spain

2. Muhlbauer MC, Kneller SK (2013) Radiography of the dog and cat: Guide to making and interpreting radiographs. $\left(1^{\text {st }} e d n\right)$, Wiley-Blackwell, USA.

3. Oliver González MR, García Tovar CG, Soto Zárate CI, Garrido Fariña G, Rodríguez Salazar LM (2017) Epistemología de la imaginación: el pensamiento geométrico en la enseñanza de la anatomía y la histología. Lat Am J Sci Educ 22115.

4. Garrosa S (1998) El concepto de dimensión. Los ejes XYZ.

5. Pérez SAP (2007) Atlas de anatomía radiográfica: órganos digestivos abdominales y tránsito gastrointestinal en perro adulto (Canis familiaris). Tesis profesional. FES-Cuautitlán UNAM.

6. Rodríguez SLM (2015) Epistemología de la Imaginación: el trabajo experimental de Ørsted. Corinter (Ed.), Capítulo, México, p. 5.

7. Evans HCE, de Lahunta A (2013) Miller's Anatomy of the Dog, (4 $4^{\text {th }}$ edn), Elsevier Saunders, USA.

8. Oliver GRS (2017) Epistemología aplicada a las ciencias biológicas básicas. 3er Congreso de Ciencia, Educación y Tecnología, Facultad de Estudios Superiores Cuautitlán (FES-C) UNAM.

9. Gasse H (2012) Nomina anatomica veterinaria. International Committee on Veterinary Gross Anatomical Nomenclature. ( $5^{\text {th }}$ edn), Editorial Committee, Hannover, Germany.
Creative Commons Attribution 4.0 International License

For possible submissions Click Here

\section{Submit Article}

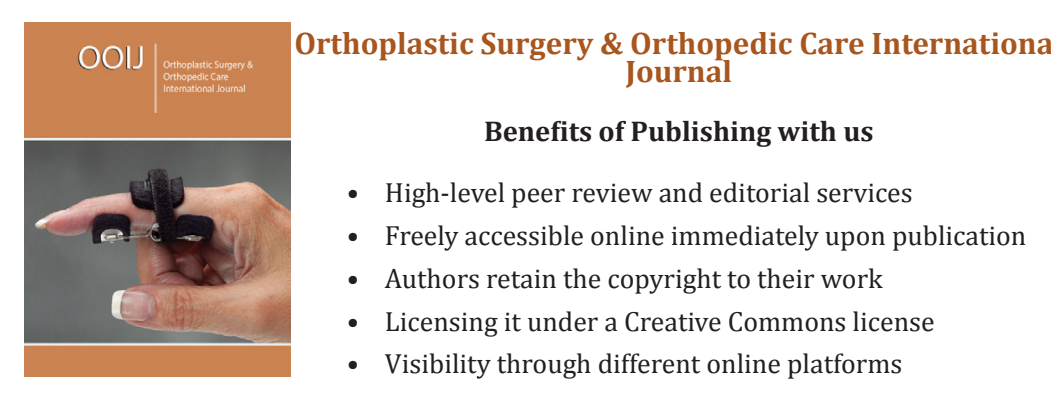

